\title{
Perspectiva histórica y estado actual de la formación Doctoral en Psicología en Argentina: el Doctorado de la Universidad Nacional de Córdoba
}

\section{Historical perspective and current status} of Doctoral training in Psychology in Argentina: the Doctorate of the National University of Córdoba

\section{Perspectiva histórica e estado atual da formação doutoral em Psicologia na Argentina: o Doutorado da Universidade Nacional de Córdoba}

\author{
Silvina Brussino* \\ Juan Carlos Godoy*
}

\begin{abstract}
RESUMEN
Este artículo tiene por objeto describir el estado actual de la formación doctoral de los psicólogos en Argentina. Para ello se realiza una primera descripción histórica sobre el desarrollo de la Psicología como disciplina científica, donde se plasman las primeras articulaciones entre investigación y formación. Luego, se particulariza sobre la aparición de los primeros laboratorios de investigación y su relación con la formación académica en psicología en Argentina, para continuar en otro apartado, con la discusión
\end{abstract}

* Universidad Nacional de Córdoba y Consejo Nacional de Investigaciones Científica y Técnicas (CONICET). Instituto de Investigaciones Psicológicas (IIPsi). Córdoba, Argentina. E-mails: silvina.brussino@unc.edu.ar.https://orcid.org/0000-0002-1087-644X; jcgodoy@conicet. gov.ar. https://orcid.org/0000-0002-1622-1647. 
actual sobre la formación de grado y posgrado en el contexto latinoamericano. Finalmente, se brinda un estado actual de la oferta de programas de doctorado en Psicología a partir de los informes del Consejo Nacional de Evaluación y Acreditación Universitaria, haciendo foco en el programa de doctorado de la Universidad Nacional de Córdoba que es el más antiguo y el que posee la mayor categoría de acreditación de calidad en el país. Se concluye con la presentación de algunos ejes que podrían guiar el debate sobre la formación doctoral en Psicología en Argentina y en Iberolatinoamérica.

Palabras claves: Formación en psicología. Doctorado en psicología. Formación en investigación. Psicología en Argentina.

\begin{abstract}
The purpose of this article is to describe the current status of doctoral training for psychologists in Argentina. To this end, a first historical description is made of the development of Psychology as a scientific discipline, where the first articulations between research and training take shape. Then, it is particularized about the appearance of the first research laboratories and their relationship with the academic training in psychology in Argentina, to continue in another section, with the current discussion on graduate and postgraduate training in the Latin American context. Finally, a current state of the offer of doctoral programs in Psychology is provided based on the reports of the National Council for University Evaluation and Accreditation, focusing on the doctoral program of the National University of Córdoba, which is the oldest and which has the highest quality accreditation category in the country. It concludes with the presentation of some topics that could guide the debate on doctoral training in Psychology in Argentina and IberoLatin America.
\end{abstract}

Keywords: Psychology training. Doctorate in psychology. Research training. Psychology in Argentina.

\title{
RESUMO
}

Este artigo tem por objetivo descrever o estado atual da formação em doutorado dos psicólogos na Argentina. Para isso, realiza-se uma primeira descrição histórica sobre o desenvolvimento da Psicologia como disciplina científica, em que se plasmam as primeiras articulações entre pesquisa e formação. Em seguida, focaliza-se sobre a aparição dos primeiros laboratórios de investigação e sua relação com a formação acadêmica em psicologia na Argentina. Na seção seguinte, continua-se com a discussão atual sobre a formação de graduação e/ou pós-graduação no contexto latino-americano. Finalmente, brinda-se com o estado atual da oferta de programas de douto- 
rado em Psicologia, tomando como base os informes do Conselho Nacional de Avaliação e Acreditação Universitária, focalizando-se no programa de doutorado da Universidade Nacional de Córdoba, que é o mais antigo e o que possui a maior nota creditada em termos de qualidade no país. Conclui-se com a apresentação de alguns eixos que poderiam guiar o debate sobre a formação ao nível de doutorado em Psicologia na Argentina e no contexto ibero-latino-americano.

Palavras-chave: Formação em psicologia. Doutorado em psicologia. Formação em pesquisa. Psicologia na Argentina.

\section{Introducción}

Se atribuye a Ebbinghaus una descripción del desarrollo de nuestra disciplina que resiste el paso del tiempo: "La psicología tiene un largo pasado, pero una historia corta". En efecto, podemos afirmar que el estudio de temas psicológicos precede al establecimiento de la psicología como una disciplina científica. Así, por caso, las investigaciones en psicofísica desarrolladas antes de 1870 por Weber, Fechner y von Helmholtz son consideradas como el primer programa de investigación psicológica. Por lo mismo, diversos investigadores de la historia de la psicología coinciden en considerar a Fechner como padre de la psicología experimental (ver, por ejemplo: LEAHEY, 1997a, p. 203-246; ATO, 1998; DEHUE, 2001). Por otro lado, además de anclar los orígenes de la psicología académica a la Psicofísica, el mismo Leahey (1997a, p. 203-246) sugiere otras tres "fundaciones" posibles de nuestra disciplina a finales del siglo XIX: una vinculada con la Psicología de la Conciencia de Wundt y otros, otra relacionada con el Psicoanálisis de Freud (1856-1939), y otra más con la Psicología de la Adaptación de James (1842-1910) y otros.

Sin embargo, existe un acuerdo generalizado en asociar los orígenes de la psicología con la creación del primer laboratorio de psicología experimental por parte de Wundt (1832-1920) en la Universidad de Leipzig, Alemania, en 1879. Esto resulta evidente al analizar diversos manuales de historia de la psicología de uso generalizado en diversas regiones (GONDRA, 1997; TORTOSA Y COLS, 1998; HERGENHAHN, 2001). Sin embargo, pocos tienen presente que ese laboratorio se inició con las investigaciones correspondientes a la tesis doctoral de M. Friedich, quien estaba interesado en el tiempo de apercepción de los procesos mentales simples y compuestos (TORTOSA Y COLS, 1998). Así, el proceso de institucionalización de la psicología tiene una deuda importante con el concepto de laboratorio y la dinámica de formación de los jóvenes recursos 
humanos que concibió Wundt, quien fungía como director y supervisaba todas las tesis doctorales. De hecho, muchos de esos jóvenes ya doctores, fueron responsables de la proliferación de otros laboratorios dedicados a estudiar los procesos psíquicos con un abordaje experimental. Así, podría afirmarse que la psicología empezó primero con un interés en la formación de posgrado: los doctores en psicología llegaron antes que los licenciados o graduados en psicología. El lector interesado en profundizar más sobre el rol de los primeros laboratorios de psicología en la institucionalización de nuestra disciplina, y en sus características, puede analizar los trabajos de K. Danziger $(1985,1990)$.

Con todo, muy rápidamente comenzaron a crearse cursos de grado de psicología tanto en Europa como en Estados Unidos. Por caso, hacia fines del siglo XIX Wundt impartía clases de psicología experimental en su laboratorio en Leipzig (Alemania), mientras que James impartía un curso de psicología fisiológica también en su laboratorio en Harvard (EE. UU.). Sin embargo, habría que esperar hasta mediados del siglo XX para llegar a ver un diseño curricular integral para la formación de los psicólogos: el modelo de formación científico-practicante (o Modelo Boulder, ver más adelante). Este programa de formación de grado en psicología resultó de los consensos logrados entre agencias gubernamentales de los EE.UU. (como la Asociación de Veteranos de Guerra) y las principales instituciones de educación superior que tenían carreras de psicología. El encuentro entre todos estos actores tuvo lugar en Boulder (Colorado, EE.UU.) en 1949 y dejó como resultado un modelo de formación de grado en psicología conformado por dos ciclos: los cursos iniciales del grado son de carácter "científico" y los cursos superiores se destinan a la formación "profesional". Esta estrategia curricular pretendía garantizar una formación adecuada tanto para ser psicólogos investigadores o académicos, como para dedicarse a las prácticas profesionales, especialmente, a la psicología clínica. Este diseño curricular de grado se desarrolla hoy en casi todo el mundo occidental (BAKER; BENJAMIN, 2000; LEAHEY, 1997b), aunque no está libre de críticas (FRANK, 1984; ALBEE, 2000). En Argentina y Latinoamérica la adopción del modelo llevó más tiempo.

\section{Los primeros laboratorios de psicología y la formación académica en psicología en Argentina}

Diversos autores coinciden en señalar que la psicología científica en Argentina tuvo su inicio a fines del siglo XIX y a poco tiempo de la creación de 
los primeros laboratorios de psicología en Europa y en EE.UU. En efecto, el primer laboratorio de psicología de Argentina fue creado en la ciudad de San Juan por Víctor Mercante (1870-1934) en 1891 (PAPINI, 1976; CORTADA DE KOHAN, 1978; KLAPPENBACH; PAVESI, 1994). Por su parte, en 1898, Horacio Piñero (1869-1919) crea un laboratorio de psicología experimental en el Colegio Nacional de Buenos Aires y, en 1901, crea un laboratorio de psicofisiología en la Universidad de Buenos Aires (PAPINI, 1976; CORTADA DE KOHAN, 1978; SÁNCHEZ-SOSA; VALDERRAMA-ITURBE, 2001). Al respecto, y marcando las primeras diferencias con los laboratorios europeos y norteamericanos, en el laboratorio creado por Piñero los alumnos no solo realizaban experimentos, sino que también trabajaban con pacientes que eran estudiados desde una perspectiva clínica.

Así, los primeros estudiantes de psicología de Argentina comenzaban su formación bajo la fuerte influencia de los trabajos de Charcot, Wundt, Ribot y Janet (CORTADA DE KOHAN, 1978). Sin embargo, la influencia de Wundt y de su programa de investigación fue casi nula en nuestro país. Al respecto, Miceli (1994) señala que en Argentina "la influencia de Ribot enlazará la psicología a la patología mental, característica de la tradición francesa, a la cual estará ligada nuestra psicología antes que a ninguna otra", impactando significativamente sobre la labor de las dos principales figuras de los inicios de la psicología en el país: Horacio Piñero y José Ingenieros. En consecuencia, la psicología científica argentina comienza su transición hacia una psicología clínica. En efecto, tanto Piñero como Ingenieros, referentes de las primeras cátedras de psicología de la Universidad de Buenos Aires, son los principales exponentes de "la tradición francesa de la doble formación médico-filosófica” (MICELI, 1994, p. 23-32).

Otros factores, sociales y políticos, también jugaron un papel importante en el incipiente desarrollo de la formación de los psicólogos en Argentina. Por caso, antes de 1930, una "reacción antipositivista" representada por Korn y Alberini (ROSSI, 1994) produjo el cierre de la mayoría de los laboratorios de investigación psicológica en Argentina (VILANOVA, 1994). En efecto, con la llegada de Coriolano Alberini (1886-1960) a la Facultad de Filosofía y Letras de la Universidad de Buenos Aires, la psicología argentina se transforma en una psicología filosófica o "psicología de sillón" (CORTADA DE KOHAN, 1978). A este hecho se suma que desde el golpe de estado de 1930 que derrocó al gobierno de Yrigoyen y hasta 1945, aproximadamente, en Argentina se impuso un "integralismo religioso" y cualquier intento de estudio científico de la mente humana perdió apoyo. Asimismo, durante el primer gobierno de Perón, en 1949 se decretó la prohibición de la enseñanza de la psicología experimental (VILANOVA, 1994). La situación de la psicología científica argentina en los comienzos de la década del 50 del siglo XX es de tal gravedad que, por ejem- 
plo, Foradori la expone en su "Historia de la Psicología en América" de 1952, alertando "con preocupación" sobre el cierre de Institutos y Laboratorios de Psicología Experimental en el país (ROSSI, 1994).

Con todo, es justamente en ese periodo que comienzan a crearse las carreras de psicología en las principales universidades públicas argentinas. En el caso de la Universidad Nacional de Córdoba, la carrera de psicología fue creada en 1958 en el seno de la Facultad de Filosofía y Humanidades. Estas primeras carreras, por cierto, no contaban con presupuestos adecuados, ni tampoco con planteles de psicólogos con formación pertinente para ejercer la docencia o para desarrollar investigaciones en la tradición de los principales centros académicos de Europa y de EE.UU. (ARDILA, 1986; CORTADA DE KOHAN, 1978; PIÑEDA, 2004; VILANOVA, 1994). En efecto, la mayoría de los docentes de las flamantes carreras de psicología de Argentina eran médicos psiquiatras, educadores, filósofos o teólogos (esto ocurrió, por ejemplo, en la Universidad Nacional de Córdoba) y, buena parte de ellos, eran clínicos sin experiencia en investigación y formados en el campo del psicoanálisis (ARDILA, 1986; PIÑEDA, 2004; VILANOVA, 1994). Por otro lado, los primeros planes de estudio de psicología en Argentina aún estaban muy lejos del diseño básico acordado en Boulder que apuntaba a una formación científico-practicante. Habrá que esperar hasta la década de 1970 para que ello comience a revertirse (ver más adelante).

\section{La formación académica de grado y de posgrado de los psicólogos en las Américas}

En la Argentina, como puede observarse, han sido varios los obstáculos para lograr una adecuada articulación entre la investigación y la práctica necesarias para una formación integral del estudiante de grado (AUAPSI, 2001; FERNÁNDEZ ACEVEDO, 2001; GODOY; PAUTASSI, 2006; VILANOVA, 2001) y del posgrado (MOLINA, 2012). Con algunas diferencias, esta situación también se ha observado en otros países, como Brasil (DIMENSTEIN, 2003; YAMAMOTO, 2004); Chile (VERA-VILLARROEL; MOYANO-DÍAZ, 2005); España (BLANCO, 1998); y Paraguay (COPPARI DE VERA, 2008, 2009).

$\mathrm{Al}$ respecto, resulta interesante destacar que hubo que esperar hasta la I Conferencia Latinoamericana sobre Entrenamiento en Psicología que tuvo lugar en Bogotá (Colombia) en 1974, para que se refrendara el modelo científico-practicante y comenzara su implementación plena, con algunas adaptaciones, en las principales universidades latinoamericanas (ALARCÓN, 2004; ARDILA, 
1978, 1986; GALLEGOS, 2010). Desde entonces, han sido diversas las ocasiones en las que se ha revisado críticamente la formación de grado y de posgrado en psicología en el ámbito Iberolatinoamericano. En ese sentido, cabe destacar el esfuerzo de la Sociedad Interamericana de Psicología visible primero, en la publicación de "Psicología en las Américas" de M. Alonso y A. Eagly en 1999; y más claramente en su importante obra colectiva "Problemas centrales para la formación académica y el entrenamiento profesional del psicólogo en las Américas" publicada en 2001 bajo la coordinación de J. P. Toro y J. F. Villegas. En el MERCOSUR también ha habido intentos por caracterizar las oportunidades y los desafíos en la formación de los psicólogos en los países que lo conforman (VILANOVA; DI DOMÉNICO, 1999).

La discusión sobre la formación en Psicología tanto en el grado como en el posgrado también ha sido central para otras asociaciones científico-académicas. Por ejemplo, en 2005, y como resultado de diversos intercambios mantenidos en el ámbito de la Federación Iberoamericana de Psicología se publicó un monográfico de la Revista Mexicana de Psicología sobre "Evaluación de la calidad y acreditación de programas de Psicología", con contribuciones de colegas de Chile, EE.UU., España y México. De interés, para este trabajo, son los artículos "La valoración de Programas de Doctorado en Psicología. Una cuestión abierta" de V. Pelechano, y "Desafios de la Formación del Postgrado en Iberoamérica en un Mundo Globalizado" de F. Martos (que retomaremos más adelante). Por su parte, también la Asociación de Unidades Académicas de Psicología (AUAPsi) encaró el tema en el marco del $1^{\text {er }}$ Encuentro de Unidades Académicas de Psicología de Gestión Pública en Latinoamérica, resultando de ello la publicación, en 2012, de la obra colectiva "La Formación en Psicología" dirigida por P. Altamirano.

\section{Los programas de Doctorado en Psicología en Argentina}

A los fines de presentar este apartado, cabe señalar que el marco regulatorio para la creación, funcionamiento y acreditación de la enseñanza de postgrado en Argentina está provisto por la Ley de Educación Superior ( $n^{\circ} 24.521$ y modificatorias). En el marco de esta ley, se establece que las carreras de postgrado deberán ser acreditadas por la Comisión Nacional de Evaluación y Acreditación Universitaria (CONEAU). Asimismo, cabe señalar que en función de la Resolución $n^{\circ}$ 160/11 del Ministerio de Educación, la formación doctoral tiene por objeto la formación de posgraduados que puedan lograr aportes originales a un 
área de conocimiento, a través de una formación que se centre fundamentalmente en torno a la investigación. Al respecto, cabe destacar otras miradas sobre la importancia de la formación doctoral. Por ejemplo, Lvovich (2009) considera que los programas doctorales son necesarios para el desarrollo científico, académico y productivo de Argentina, tanto como para el mejoramiento de las capacidades de gestión estatal del país.

En base a una revisión realizada sobre los datos publicados por la Comisión Nacional de Evaluación y Acreditación de Universitaria (CONEAU, 2018), existen a la fecha trece (13) carreras de Doctorado en Psicología con resolución de acreditación en Argentina: diez (10) bajo la denominación genérica de Doctorado en Psicología; y las restantes con orientaciones específicas, un (1) Doctorado en Psicología con Mención en Integración Cognitivo - Existencial, un (1) Doctorado en Psicología con Orientación en Neurociencia Cognitiva Aplicada y un (1) Doctorado en Psicología Social.

Por otra parte, como se puede apreciar en el Gráfico 1, el 54\% de estos programas de posgrado (7), refieren a carreras de gestión pública. Es decir, se enmarcan en Universidades Nacionales de Argentina que se encuentran distribuidas por todo el territorio nacional (de Córdoba, de Buenos Aires, de San Luis, de La Plata, de Mar del Plata, de Rosario y de Tucumán). En tanto, las restantes carreras dependen de Universidades de gestión privada y todos ellas están radicadas en la Ciudad Autónoma de Buenos Aires (Universidad del Salvador, Universidad de Ciencias Empresariales y Sociales, Universidad de Flores, Universidad Maimónides y Universidad Argentina John F. Kennedy).

En el mismo gráfico se plasman los años de creación de los programas de Doctorado de Argentina. Sin lugar a dudas, la Universidad Pública es pionera en la creación de este tipo de programas de formación superior, y la mayor parte (54\%) se creó a partir de la década de 1990. En tanto, el programa más antiguo es el de la Universidad Nacional de Córdoba (1958). Este crecimiento es coincidente con lo planteado por Lvovich (2009) quien sostiene que, en los últimos 15 años, la oferta de posgrado en Argentina ha crecido de manera significativa, aunque sin planificación lo que lleva a un sistema sumamente heterogéneo en cuanto a estructura y calidad. Al respecto, Palacio (2005) sostiene que los doctorados tienen una presencia tardía en el marco de la educación superior en América Latina y el Caribe. Sólo el 13\% de poco más de dos mil universidades de la región tienen capacidad para formar doctores e investigadores; y buena parte de esta oferta se concentra en Brasil, México, Chile, Cuba, Argentina y Venezuela. 


\section{GRÁFICO 1: AÑO DE CREACIÓN DE LOS PROGRAMAS DE DOCTORADO SEGÚN TIPO DE GESTIÓN (PÚBLICA/PRIVADA)}

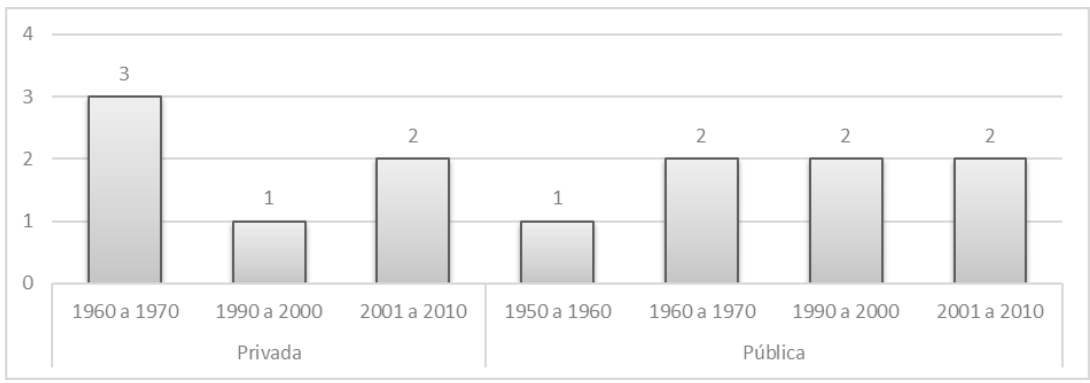

Gráfico de elaboración propia.

FUENTE: CONEAU (2018).

Tomando en consideración el eje de calidad de los programas de formación, en el Gráfico 2 se pueden advertir las diferencias que existen entre los programas de doctorado en base a las categorizaciones obtenidas por parte de la Comisión de Acreditación de la CONEAU. Las carreras de universidades públicas poseen mejores categorizaciones: $46 \%$ poseen categoría A y B, en tanto solo el 8\% de las privadas posee estas categorizaciones.

GRÁFICO 2: PROGRAMAS DE DOCTORADO SEGÚN TIPO DE GESTIÓN (PÚBLICA/PRIVADA) SEGÚN CATEGORÍA DE ACREDITACIÓN POR CONEAU

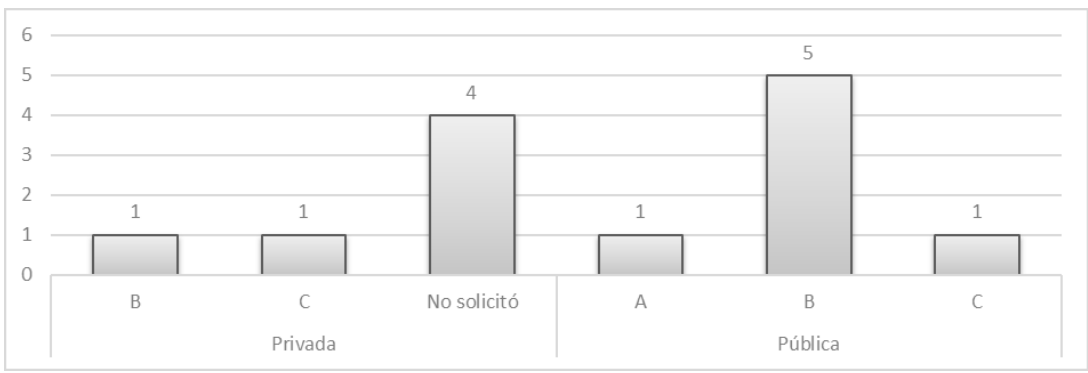

Gráfico de elaboración propia.

FUENTE: CONEAU (2018).

Aclaración Eje Categoría de Acreditación: A: si son consideradas excelentes, B: si son consideradas muy buenas, C: si son consideradas buenas y No solicitó: son carreras acreditadas, pero no categorizadas.

En cuanto a modalidad en la que se organiza el plan de estudios (Resolución $n^{\circ}$ 160/2011 Ministerio de Educación), la formación doctoral en Argentina ha 
tomado dos modalidades: a) programas personalizados, donde no se incluyen actividades curriculares preestablecidas, las mismas se definen en función del objeto de estudio de la tesis; $y$ b) programas semiestructurados, donde se ofrece un número mínimo de actividades curriculares obligatorias comunes a todos los doctorandos y un trayecto orientado en función del tema o área de estudio del trabajo de tesis. En el Gráfico 3 queda evidenciado que los Doctorados de Universidades Públicas priorizan el carácter personalizado del plan de estudios. La ausencia de planes totalmente estructurados obedece a la dificultad de englobar, en un único plan de estudios, la pluralidad de objetos que caracterizan a la Psicología, dada la diversidad de perspectivas teóricas, metodológicas y campos de aplicación que la definen. Cabe señalar que este matiz es recurrente en Argentina (HORAS, 1971; VEZZETTI, 1998) y en otras regiones (CAPARRÓS, 1991; RIBES, 2009; VILA, 2009). Por otro lado, un denominador común en todos estos planes de doctorado, es la determinación del cursado obligatorio de cursos metodológicos y/o vinculados con el análisis de datos, lo que quizá refleje la influencia del modelo científico-practicante.

\section{GRÁFICO 3: TIPO DE PLAN DE ESTUDIOS SEGÚN TIPO DE GESTIÓN (PÚBLI-} CA/PRIVADA)

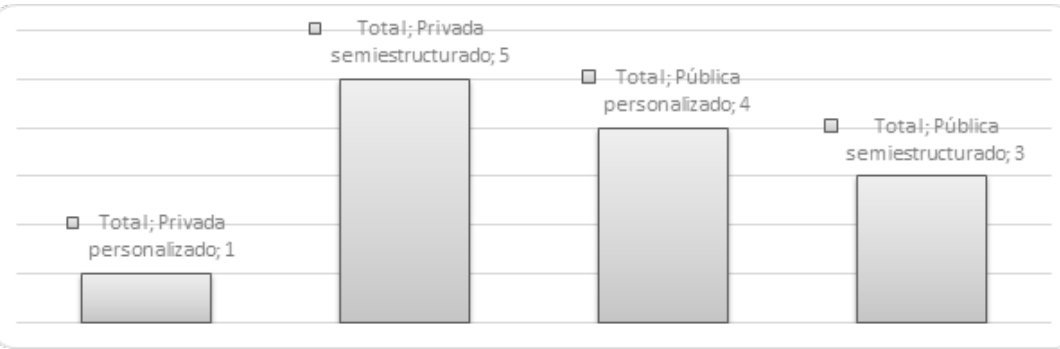

Gráfico de elaboración propia.

FUENTE: CONEAU (2018).

Por último, en cuanto al acceso a financiamiento, tal como lo señala Lvovich (2009), en Argentina existe ausencia de un financiamiento público sistemático, lo que provoca que los posgrados deban costearse mayoritariamente con los aranceles de los estudiantes. En este sentido, para financiar su formación de posgrado los estudiantes de doctorado de Universidades Públicas pueden acceder a sistemas de becas de las mismas universidades, principalmente en la Universidad de Buenos Aires y en la Universidad Nacional de Córdoba (CONEAU, 2018); y a becas que otorgan agencias nacionales como la Agencia Nacional de Promoción Científica y Tecnológica (ANPCYT), el Consejo Nacional de Investigaciones Científicas y Técnicas (CONICET) y agencias nacionales y 
provinciales, aunque estas se encuentran concentradas en las universidades de centros urbanos más importantes (Universidad de Buenos Aires, Universidad Nacional de Córdoba, Universidad de la Plata). Asimismo, cabe señalar que algunos de estos doctorados poseen un costo de matriculación mínimo, aspecto que no es equivalente el sistema privado.

En síntesis, en Argentina, tal como lo plantea Barsky y Dávila (2004) los estudios de posgrado conforman un conjunto heterogéneo de carreras ofrecidas por instituciones educativas (universidades e institutos universitarios) de gestión estatal y privadas, lo que aporta diferencias en cuanto a su origen, tradición, estructura y calidad.

\section{El doctorado en Psicología de la Universidad Nacional de Córdoba}

Tal como surge del análisis del apartado anterior, la carrera de doctorado en Psicología de la Universidad Nacional de Córdoba (UNC) es la más antigua $\mathrm{y}$, hasta la fecha, la única con la máxima categoría de calidad (Categoría "A" según Resolución RESFC-2017-232-APN-CONEAU\#ME 13/06/2017). Tiene sus orígenes en la Facultad de Filosofía y Humanidades (1946), aunque por esa época no se otorgara un título con tal denominación. Es a partir del año 1953 que se organizan los estudios de la Facultad de Filosofía y Humanidades en cuatro Escuelas y en cada una de ellas se dictaba sus respectivas carreras: Filosofía, Letras, Historia y Pedagogía, otorgándose títulos de Licenciado, Profesor y Doctor. Por el año 1956 se transforma la Escuela de Pedagogía en la Escuela de Psicología y Pedagogía la cual otorgaba el título de Doctor en Psicología y Pedagogía. Finalmente, en 1958 se crean y organizan de manera independiente las carreras de Psicología y de Pedagogía y Psicopedagogía otorgándose títulos de Doctor en Psicología y de Doctor en Pedagogía y Psicopedagogía.

Durante el Proceso Cívico-Militar en Argentina, en los años 70 y hasta mediados de los 80 del siglo XX se dictaron normativas tanto a nivel decanal como rectoral de la UNC que reglamentaron y modificaron las carreras de Doctorado en la Facultad de Filosofía, teniendo como consecuencia la desaparición del Doctorado en Psicología. Es con la vuelta de la democracia que, en el año 1984, por Ordenanza del Honorable Consejo Superior de la UNC, se deroga toda la reglamentación vigente hasta dicha fecha sobre el otorgamiento de títulos de Doctor y se estipula que cada Facultad establecerá las normas para el otorgamiento de dicho título, en acuerdo al art. 72 del Estatuto Universitario modificado por la Ord. del HCS 17/84. En consecuencia, la Facultad de Filosofía y Humanidades 
reglamenta sus carreras de Doctorado entre las que se encuentra el Doctorado en Psicología, mediante la Res. 1044/85. Se aprueba la nueva reglamentación de las Carreras de Doctorado, la cual con las modificatorias de junio de 2000 - Ord. $\mathrm{N}^{\circ}$ 1/00 aprobada por Res. 276/00- da al Doctorado en Psicología una estructura académica propia en el marco de la recientemente creada Facultad de Psicología.

En ese sentido, un aspecto distintivo del Doctorado en Psicología de la UNC, refiere a su vinculación con los organismos de Ciencia y Técnica de Argentina. En efecto, el cuerpo académico del doctorado (que comprende a los miembros del Comité Académico, del Comité Asesor y a los Directores de Tesis) se encuentra conformado por un 54\% de investigadores del CONICET. Todos ellos, a su vez, reciben financiamiento del CONICET, la ANPCYT, y de Agencias Nacionales y Provinciales para el desarrollo de sus investigaciones. Muchos de estos organismos, por su parte, reciben a un gran número de doctorandos. Asimismo, vale destacar que este conjunto de académicos posee una alta tasa de producción científica en los últimos 10 años (con una media de 3,2 artículos por año en revistas indexadas).

Los ingresantes al Doctorado en Psicología de la UNC, desde el año 2006 hasta el año 2017, han sido 135, de los cuales se han graduado 34 sobre 37 que deberían haberse graduado según la duración oficial del doctorado (5 años). De este modo, este programa presenta una de las tasas de egresos más elevadas de los doctorados en Psicología de Argentina (92\%). Por otro lado, cabe destacar que un contexto internacional caracterizado por la baja presencia de mujeres en los ámbitos académicos y científicos (WILLIAMS, 2018), el 77\% del alumnado del Doctorado en Psicología de la UNC son mujeres. En los últimos años, además, el doctorado ha comenzado a recibir alumnos de países de la región de tal manera que a la fecha el $91 \%$ de los alumnos son argentinos, $6 \%$ son uruguayos y $3 \%$ son chilenos.

Con relación a las fuentes de financiamiento, la Carrera de Doctorado en Psicología de la UNC tiene una matrícula anual mínima (menor a los U\$S50 anuales) y es gratuita para docentes de la UNC, aunque no otorga becas propias. La mayoría de los doctorandos (el 74\%) poseen becas de organismos como la SECyT de la Universidad Nacional de Córdoba, el CONICET, la Agencia de Promoción Científica y Tecnológica, entre otros organismos de CyT (ver Gráfico 4). Asimismo, en este gráfico se puede advertir un crecimiento sostenido de las cohortes de ingresantes, con un incremento considerable entre los años 2013-2016 y una aparente caída en 2017. Estos datos revelan la sinergia entre el Doctorado y los organismos de CyT. En particular, es posible que la caída observada en el 2017 refleje el impacto producido por las políticas de ajuste y recorte presupuestario llevadas adelante por el Sistema Científico Nacional (el CONICET, en particular) y las Universidades Nacionales. 


\section{GRÁFICO 4: EVOLUCIÓN DE LAS COHORTES Y CANTIDAD DE DOCTORAN- DOS CON BECAS}

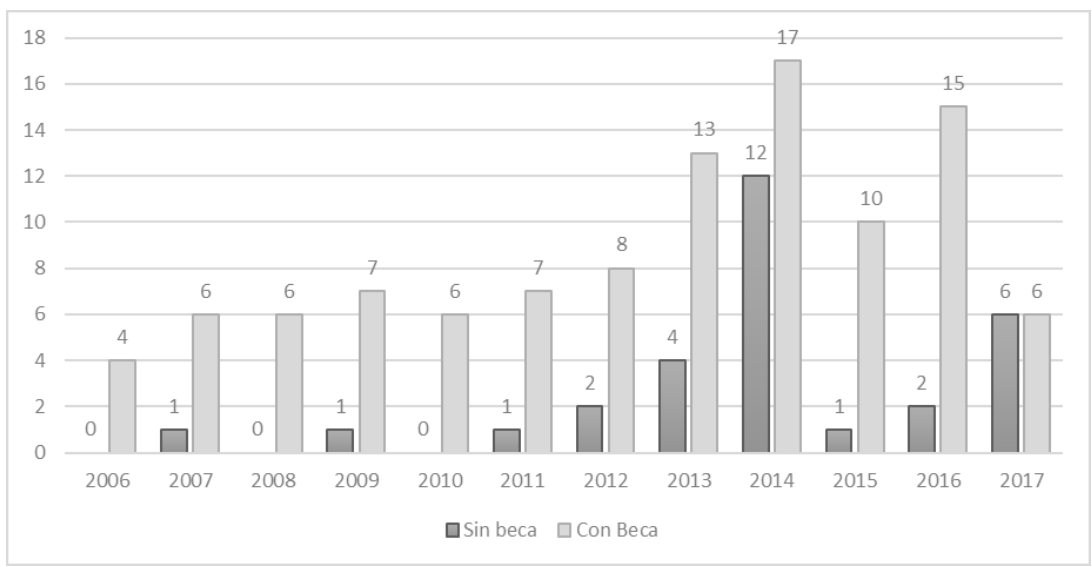

Gráfico de elaboración propia.

FUENTE: Carrera de Doctorado, Facultad de Psicología, Universidad Nacional de Córdoba (2018).

\section{Consideraciones finales}

La formación doctoral en Psicología en Argentina enfrenta muchos desafíos. Algunos de ellos son locales y, por lo tanto, requieren de articulaciones pertinentes entre los principales actores implicados: las universidades, los organismos de CyT, los organismos de acreditación, los gobiernos provinciales y nacionales, y los colegios profesionales. En este sentido, varios de esos desafíos son de larga data (COUREL; TALAK, 2001) y cuentan con una capa extra de complejidad en un contexto dominado por políticas neoliberales que pretenden que la educación, como derecho humano y como bien público, ceda terreno ante las políticas del mercado. Con todo, al menos las unidades académicas de psicología de gestión pública de Argentina se han manifestado en defensa de la Declaración mundial sobre la educación superior en el siglo XXI: visión y acción de la UNESCO y de otros documentos similares producidos en los últimos años, como queda de manifiesto en la Declaración de Montevideo, suscripta en 2012 en el marco del 1er. Encuentro de Unidades Académicas de Psicología de Gestión Pública en Latinoamérica: 
Pretendemos que nuestras facultades e instituciones formadoras contribuyan activamente a la constitución de espacios internacionales de intercambio y organización en lo que respecta a la formación disciplinaria en América Latina y el Caribe. Acompañando siempre los procesos de integración regional que nuestros estados lleven adelante, y fortaleciendo el principio de considerar a la Educación Superior como un bien público y social, un derecho humano y universal y un deber del Estado (ALTAMIRANO, 2012, p. 24-25).

Otros problemas son compartidos con otros países, después de todo, estamos en un mundo globalizado (MARTOS, 2005; PELECHANO, 2005). Así, entre los desafíos que marcan la agenda compartida entre los diferentes programas de formación doctoral de Iberolatinoamérica encontramos los derivados: de la internacionalización del conocimiento, de los esfuerzos de cooperación entre países, de la necesidad de incrementar el número de doctores y de garantizar su inserción efectiva, de la búsqueda de estándares compartidos para la acreditación de la calidad de los programas de doctorado, de los ajustes de los ciclos formativos en los programas de doctorado apuntando tanto a los perfiles académicos como a los profesionales, de la incorporación de las nuevas tecnologías como las plataformas de enseñanza virtual, y de una formación integral que también contemple la interdisciplinariedad. Por cierto, estos problemas no son los únicos que enfrentamos si queremos mejorar la formación doctoral en psicología en Argentina y en Iberolatinoamérica. Pero, son los que ofrecen más puntos en común y, por lo mismo, los que mejor garantizan la búsqueda de soluciones de manera conjunta en una región que cuenta cada vez más, con menos tiempo y menos recursos, para garantizar el bienestar de sus ciudadanos.

\section{REFERENCIAS}

ALARCÓN, R. Medio siglo de Psicología Latinoamericana: Una visión de conjunto. Revista Interamericana de Psicologia, 38, p. 307-316, 2004.

ALBEE, G. W. The Boulder model's fatal flaw. American Psychologist, 55(2), 247-248, 2000.

ALONSO, M.; M. Y EAGLY, A. Psicología en las Américas. Caracas, Venezuela: Sociedad Interamericana de Psicología (SIP), 1999. 
ALTAMIRANO, P. (Dir.) La Formación en Psicología. $1^{\text {er }}$. Encuentro de Unidades Académicas de Psicología de Gestión Pública en Latinoamérica. Córdoba: Encuentro Grupo Editor, 2012

ARDILA, R. La profesión del psicólogo. México: Trillas, 1978.

ARDILA, R. La psicología en América Latina. Pasado, Presente y Futuro. México: Siglo Veintiuno Editores, 1986.

ATO, M. Metodología de la investigación en psicología: perspectiva histórica. En: Tortosa, F. (coord.) Una historia de la psicología moderna. Madrid: McGraw Hill, p. 371-387, 1998.

AUAPSI. Actas y Declaraciones de la Asociación de Unidades Académicas de Psicología y Uruguay. Buenos Aires: Facultad de Psicología de la Universidad de Buenos Aires, 2001.

BAKER, D. B.; BENJAMIN Jr., L. T. The affirmation of the scientist-practitioner. A look back at Boulder. American Psychologyst, 55, p. 241-247, 2000.

BARSKY, O.; DÁVILA, M. Las tendencias actuales de los posgrados en Argentina, documento de trabajo n. 117, Buenos Aires, Universidad de Belgrano, 2004.

BLANCO, A. Requisitos y necesidades de formación para la psicología del siglo XXI. Revista de Psicología General y Aplicada, vol. 51, n. 1, p. 149-172, 1998.

CAPARRÓS, A. Crisis de la psicología: ¿singular o plural? Aproximación a algo más que un concepto historiográfico. Anuario de Psicología/The UB Journal of Psychology, n. 51, p. 5-20, 1991.

CONEAU. Disponible en: < http://www.coneau.gov.ar/buscadores/posgrado/buscar.php 2018>. Acceso: 15 mar. 2018.

COPPARI de VERA, N. Psicología. Una sola Ciencia, diversas Artes. Desafíos para definir y Acreditar la Carrera en nuestro país y en el MERCOSUR. Eureka, n. 5, p. 111-121, 2008.

COPPARI de VERA, N. Psicología. Desafíos para Definir y Acreditar la Carrera en nuestro país y en el MERCOSUR. Eureka, n. 6, p. 119-134, 2009.

CORTADA DE KOHAN, N. La psicología en Argentina. En: Ardila, R. (coord.) La profesión del psicólogo (pp. 30-43). México: Editorial Trillas, 1978.

COUREL, R.; TALAK, A. M. La formación académica y profesional del psicólogo en Argentina. En: Toro, J., \& Villegas, J. (comps.). Problemas centrales para la formación académica y el entrenamiento profesional del psicólogo en las Américas. Buenos Aires, Argentina: Sociedad Interamericana de Psicología (SIP), 2001, Vol. 1, pp. 21-83.

DANZIGER, K. Historical roots of the psychological laboratory. In Constructing the Subject: Historical Origins of Psychological Research. Cambridge: Cambridge University Press, 1990, pp. 17-33. doi:10.1017/CBO9780511524059.003. 
DANZIGER, K. Origins of the psychological experiment as a social institution. American Psychologist, 40, 133-140, 1985.

DEHUE, T. History of experimentation in psychology. En: International Encyclopedia of the Social \& Behavioral Sciences, Elsevier Science Ltd, p. 5115-5120, 2001.

DIMENSTEIN, M. Los (des)caminos de la formación profesional del psicólogo en Brasil para la actuación en la salud pública. Revista Panamericana de Salud Pública, n. 13 , p. 341-345, 2003.

FERNÁNDEZ ACEVEDO, G. La formación en investigación de los psicólogos en el grado universitario en la Argentina. Acta Psiquiátrica y Psicológica de América Latina, v. 47, n. 1, p. 39-50, 2001.

FRANK, G. The Boulder Model: History, rationale, and critique. Professional Psychology: Research and Practice, v. 15, n. 3, p. 417, 1984.

GALLEGOS, M. La primera Conferencia Latinoamericana sobre Entrenamiento en Psicología (1974) el modelo Latinoamericano y su significación histórica. Psicologia Ciência e Profissão, v. 30, n. 4, p. 792-809, 2010. Disponible en: <https//dx.doi.org10. 1590S1414-98932010000400010>. Acceso: 29 de marzo de 2018.

GODOY, J. C.; PAUTASSI, R. M. Relación entre la investigación básica y las prácticas profesionales en la psicología contemporánea. En: Godoy, J. C. (comp.) Investigaciones históricas en Psicología. Córdoba, Universidad Nacional de Córdoba, p. 151-163, 2006.

GONDRA, J. M. Historia de la Psicología. Introducción al pensamiento psicológico moderno. Vol. 1: Nacimiento de la psicología científica. Madrid, Editorial Síntesis, p. 89-121, 1997.

HERGENHAHN, B. R. Primeras aproximaciones a la Psicología. En: Hergenhahn, B. R. Introducción a la Historia de la Psicología. Madrid, Paraninfo, p. 271-303, 2001.

HORAS, P. ¿Una o varias psicologías? Intento de integración. En Actas del Segundo Congreso Argentino de Psicología. San Luis. Facultad de Pedagogía y Psicología de la Universidad Nacional de Cuyo, p. 145-150, 1971.

KLAPPENBACH, H.; PAVESI, P. Una historia de la Psicología en Latinoamérica. Revista Latinoamericana de Psicología, n. 26, p. 445-482, 1994.

LEAHEY, T. H. Los umbrales de la Psicología en el Siglo XIX. En: LEAHEY, T. H. Historia de la Psicología. Corrientes principales del pensamiento psicológico. Madrid, Debate, 1997. pp. 203-246.

LEAHEY, T. H. La Psicología alza el vuelo: Psicología y Sociedad (1913-1950). En: LEAHEY, T. H. Historia de la Psicología. Corrientes principales del pensamiento psicológico. Madrid, Debate, p. 445-488, 1997 b.

LVOVICH, D. Resultados e impactos de los programas de apoyo a la formación de posgrado en Argentina. Revista CTS, v. 13, n. 5, p. 157-173, 2009. 
MARTOS, F. J. Desafíos de la formación del postgrado en Iberoamérica en un mundo globalizado. Revista mexicana de Psicología, n. 22, p. 333-344, 2005.

MICELI, C. El método patológico de Ribot y la psicología experimental en la Argentina de principios de siglo. En: Rossi, L. (Coord.) Psicología en Argentina. Capítulos olvidados de una historia reciente. Buenos Aires, Editorial Tekné, p. 23-32, 1994.

PALACIO, J. L. Tendencias de desarrollo de los programas de postgrado en América Latina y el Caribe. Contexto y situación actuales. Salamanca, Ediciones AUIP, 2005.

PAPINI, M. Datos para una historia de la Psicología Experimental Argentina, hasta 1930. Revista Latinoamericana de Psicología, n. 8, p. 319-335, 1976.

PELECHANO, V. La valoración de programas de doctorado en Psicología: una cuestión abierta. Revista mexicana de Psicología, n. 22, p. 327-332, 2005.

PIÑEDA, M. A. Comienzos de la profesionalización de la psicología, la Universidad Nacional de Córdoba y el movimiento neoescolástico. Memorandum, n.7, pp. 165-188, 2004. Recuperado el 24/03/08 de World Wide Web: http://www.fafich.ufmg.br/ memorandum/ $\operatorname{artigos} 07 /$ pineda01.htm

RIBES, E. La psicología como ciencia básica. ¿Cuál es su universo de investigación? Revista Mexicana de Investigación en Psicología, v. 1, n. 2, p. 7-19, 2009.

ROSSI, L. Introducción. Contexto de creación de las Carreras de Psicología. En: Rossi, L. (Coord.) Psicología en Argentina. Capitulos olvidados de una historia reciente. Buenos Aires: Editorial Tekné, p. 11-22, 1994.

SÁNCHEZ-SOSA, J. J.; VALDERRAMA-ITURBE, P. Psychology in Latin America: Historical reflections and perspectives. International Journal of Psychology, n. 36, p. 384-394, 2001.

TORO, J.; VILLEGAS, J. (comps.) Problemas centrales para la formación académica y el entrenamiento profesional del psicólogo en las Américas (Vol. 1). Buenos Aires, Argentina: Sociedad Interamericana de Psicología (SIP), 2001.

TORTOSA, F.; PASTOR, J. C.; QUINTANA, J.; SÁIZ, M.; SÁIZ, D.; MÜLBERGER, A. El nacimiento de la psicología académica en Alemania: La psicología "moderna" hasta 1910. En: Tortosa, F. (coord.) Una historia de la psicología moderna. Madrid: McGraw Hill, p. 97-120, 1998.

VERA-VILLARROEL, P.; MOYANO-DÍAZ, E. La Acreditación de los Programas de Psicología en Chile: Antecedentes Contextuales y Estado Actual. Revista Mexicana de Psicología, n. 22, p. 293-305, 2005.

VEZZETTI, H. 1998. Las psicologías del fin de siglo a la luz de su historia. Revista de psicología general y aplicada, v. 51, n. 1, p. 105-114, 1998.

VILA, J. Sobre las otras psicologías. Revista Mexicana de Investigación en Psicología, v. 1, n. 2, p. 38-40, 2009. 
VILANOVA, A. La investigación psicológica en la Argentina. Nexos, Año 1, Vol. 1, 1994. Secretaría de Ciencia y Técnica de la Universidad Nacional de Mar del Plata. Reimpreso en: Vilanova, A. Discusión por la Psicología. Departamento de Servicios Gráficos: Universidad Nacional de Mar del Plata, p. 44-47, 2003.

VILANOVA, A. La formación académica del psicólogo en el mundo y en Argentina. Acta Psiquiátrica y Psicológica de América Latina, n. 47, p. 73-78, 2001.

VILANOVA, A. La investigación psicológica en la Argentina. Nexos, Secretaría de Ciencia y Técnica UNMdP, año 1, n. 1, 1994. Reimpreso en Vilanova, A. (2003) Discusión por la psicología (pp. 44-47). Mar del Plata: Departamento de Servicios Gráficos, UNMdP. ISBN 987-544-084-1.

VILANOVA, A.; DI DOMÉNICO, C. La Psicología en el Cono Sur. Datos para una historia. Mar del Plata: Editorial Martín, 1999.

WILLIAMS, W. M. Underrepresentation of women in science: international and crossdisciplinary evidence and debate. Frontiers in Psychology, n. 8, p. 2352, 2018.

YAMAMOTO, O. H. Reforma del Estado, Ajuste Neoliberal y Políticas Educacionales: la Formación en Psicología en Brasil. Revista Interamericana de Psicología, n. 38, p. 191-199, 2004.

Texto recibido en 17 de mayo de 2018. Texto aprobado en 17 de julio de 2018. 